\title{
New therapeutic strategies for the treatment of male lower urinary tract symptoms
}

This article was published in the following Dove Press journal:

Research and Reports in Urology

26 April 2016

Number of times this article has been viewed

\section{Konstantinos Dimitropoulos Stavros Gravas \\ Department of Urology, Faculty of Medicine, School of Health Sciences, University of Thessaly, Larissa, Greece}

Correspondence: Stavros Gravas Department of Urology, Faculty of Medicine, School of Health Sciences, University of Thessaly, 6-8 Feidiou, Larissa $4 \mid 22$ I, Greece

Tel +306944626086

Email sgravas2002@yahoo.com
Abstract: Male lower urinary tract symptoms (LUTS) are prevalent in the general population, especially in those of advanced age, and are characterized by notable diversity in etiology and presentation, and have been proven to cause various degrees of impairment on quality of life. The prostate has traditionally been regarded as the core cause of male LUTS. As a result, medical treatment aims to provide symptomatic relief and effective management of progression of male LUTS due to benign prostatic enlargement. In this context, $\alpha_{1}$-blockers, phosphodiesterase- 5 inhibitors, and $5 \alpha$-reductase inhibitors have long been used as monotherapies or in combination treatment to control voiding LUTS. There is accumulating evidence, however, that highlights the role of the bladder in the pathogenesis of male LUTS. Current research interests have shifted to bladder disorders, and medical management is aimed at the bladder. Muscarinic receptor antagonists and the newly approved $\beta_{3}$-adrenergic agonist mirabegron aim to alleviate the most bothersome storage LUTS and thus improve quality of life. As voiding and storage LUTS frequently coexist, combination therapeutic strategies with $\alpha_{1}$-blockers and antimuscarinics or $\beta_{3}$-agonists have been introduced to manage symptoms effectively. Anti-inflammatory agents, vitamin $\mathrm{D}_{3}$-receptor analogs, and cannabinoids represent treatment modalities currently under investigation for use in LUTS patients. Furthermore, luteinizing hormone-releasing hormone antagonists, transient receptor-potential channel blockers, purinergic neurotransmission antagonists, Rho-kinase inhibitors, and inhibitors of endothelin-converting enzymes could have therapeutic potential in LUTS management, but still remain in the experimental setting. This article reviews new strategies for the medical treatment of male LUTS, which are dictated by the potential role of the bladder and the risk of benign prostatic hyperplasia progression. Moreover, combination treatments and therapies currently under investigation are also presented.

Keywords: medical treatment, lower urinary tract symptoms, benign prostatic hyperplasia, overactive bladder syndrome

\section{Introduction}

Male lower urinary tract symptoms (LUTS) are characterized by notable diversity in presentation, and according to their characteristics they are classified into three general categories: voiding, storage, and post-micturition symptoms. ${ }^{1}$ The etiology of male LUTS is multifactorial, and prostatic, bladder, and other urinary tract disorders, as well as nonurological conditions, have been proposed to be implicated in the pathogenesis of LUTS in males while research is ongoing to reveal other potential pathogenetic pathways. ${ }^{1-3}$ Nevertheless, it is known that LUTS are prevalent in the general population, as a large multinational study revealed that approximately two-thirds of males 
report at least one LUTS complaint during their lifetime. They are directly related to the aging process, and influence patients' lives to various degrees. ${ }^{4-6}$

The prostate has traditionally been regarded as the core of male LUTS, and thus physicians were routinely directed toward use of prostate-targeted treatment modalities for managing symptoms, mainly $\alpha_{1}$-blockers and $5 \alpha$-reductase inhibitors ( $5 \alpha \mathrm{RIs}) .^{7}$ However, there is growing evidence that questions the role of the prostate as the main pathogenetic cause of male LUTS. It has been shown that LUTS are not frequently in males only; a high prevalence of LUTS has also been described in females, mainly due to overactive bladder (OAB). ${ }^{6}$ Also, not all males with benign prostatic enlargement (BPE) report LUTS, and not all male LUTS patients suffer from bladder-outlet obstruction (BOO) due to BPE, as OAB has been proved to be common in males too. ${ }^{8,9}$ In addition, surgical treatment of BOO due to BPE resulting from benign prostatic hyperplasia $(\mathrm{BPH})$ has not been shown to lead to significant regression of symptoms in all patients. ${ }^{10,11}$

Nowadays, the role of the prostate as the core of male LUTS is currently under dispute, and the bladder has become the new target for research. Accumulating evidence shows that bladder dysfunction is directly involved in the development of symptoms, and as such managing bladder disorders could mean control of LUTS. ${ }^{12,13}$ On the other hand, there is special attention being paid to the management of progression of male LUTS due to BPE. Clinical progression of BPH events (defined as an International Prostate Symptom Score [IPSS] increase $\geq 4$ points, acute urinary retention [AUR], urinary incontinence, renal insufficiency, or recurrent urinary tract infections) had a cumulative incidence of 17\% among males in the placebo arm of the Medical Therapy of Prostatic Symptoms (MTOPS) study, who had follow-up data of at least 4 years. ${ }^{14}$ Although AUR and surgery are less common than overall symptomatic worsening, they represent important events of disease progression, because of the financial, emotional, and health-related implications, and thus represent the major concerns of BPH patients. This article reviews new strategies for the medical treatment of male LUTS, which are dictated by the potential role of the bladder and the risk of BPH progression. Moreover, combination treatment and therapies currently under investigation are also presented.

\section{Currently used treatment modalities $\alpha_{1}$-Blockers}

According to current evidence, blockage of $\alpha_{1}$-adrenoceptors can offer an improvement of about $30 \%-40 \%$ on both storage and voiding symptoms, though without affecting the progression of $\mathrm{BPH}$, as $\alpha_{1}$-blockers fail to reduce prostatic volume or risk for UR. ${ }^{14,15}$ Interestingly though, urodynamic findings have failed to show a significant effect of $\alpha_{1}$-blockers on bladder-outlet resistance, and thus the direct correlation between obstruction relief resulting from $\alpha_{1}$-blocker use and symptomatic improvement is disputed. ${ }^{16,17}$ Nevertheless, as $\alpha_{1}$-blockers offer fast and sufficient improvement in symptoms combined with a safe profile, they can be offered to males with moderate-to-severe male LUTS, according to current European Association of Urology (EAU) guidelines. ${ }^{18}$

\section{$5 \alpha$-reductase inhibitors}

After 6-12 months of 5 $\alpha$ RI treatment in patients with BPEassociated $\mathrm{BOO}$, prostate size is reduced by approximately $18 \%-28 \%$, circulating prostate-specific antigen (PSA) levels drop by $50 \%$, and maximum urinary flow $\left(\mathrm{Q}_{\max }\right)$ is increased by $1.5-2.0 \mathrm{~mL} / \mathrm{s}^{19-21}$ Reduction in symptoms offered by $5 \alpha \mathrm{RIs}$ is greater in higher baseline prostate volumes. ${ }^{18} \mathrm{In}$ contrast with $\alpha 1$-blockers, $5 \alpha$ RIs have been proved to mediate BPH progression, as they reduce episodes of AUR and the need for BPH-related surgical operations in comparison with placebo. ${ }^{22}$ In general, $5 \alpha \mathrm{RI}$ use is well tolerated, and the main adverse events comprise of disorders in sexual function. ${ }^{19} 5 \alpha$ RIs can be offered to males at risk for BPH progression (males with moderate-to-severe LUTS and prostate size $>40 \mathrm{~mL}$ and/or elevated PSA concentration, defined as $>1.4-1.6 \mathrm{ng} / \mathrm{mL}$ ).

\section{Muscarinic receptor antagonists}

While LUTS are generally divided into storage, voiding, and post-micturition symptoms, it is not unusual for males to report symptoms from more than one category. It has been shown that approximately one in three males with LUTS will report symptoms from all three LUTS categories. ${ }^{23}$ Nevertheless, most males report voiding LUTS, traditionally attributed to prostatic disorders, as their prevalence has been shown to be the highest of all. However, OAB-associated storage LUTS have been proved to be the symptoms that affect patients' lives the most. ${ }^{24}$

Even with this knowledge, first-line drugs commonly prescribed to males with LUTS remain $\alpha_{1}$-blockers and $5 \alpha$ RIs, either as monotherapies or combination treatment. It is possible that urologists primarily aim to control the prevalent voiding symptoms arising from obstruction due to PE. However, these agents may undertreat the most bothersome storage symptoms, and as a result, patients' quality of life (QoL) remains problematic. A reasonable approach 
would be to control the storage component of LUTS by reducing spontaneous detrusor activity in the storage phase. Antimuscarinic action is based on blocking acetylcholinemediated human bladder contraction. Muscarinic receptor antagonists intervene in the storage phase of the bladder state, by decreasing the frequency and intensity of bladder-muscle contractions. Moreover, antimuscarinics have been shown to inhibit bladder-afferent mechanisms during filling and thus, increase bladder capacity. ${ }^{25}$

Interestingly though, urologists seem to be reluctant in using antimuscarinics in males with BPE, due to a common fear that induced lower detrusor-voiding pressure would lead to increasing amounts of postvoid residual (PVR) or worse to AUR. ${ }^{26}$ Studies evaluated treatment with antimuscarinics in males with BOO reporting predominantly storage LUTS, and significant symptomatic improvement was shown without clinically significant increases in PVR or AUR rates. ${ }^{27,28}$ However, these trials have been criticized for their relatively short duration and for enrolling highly selected patients with low baseline prostate volumes and PVRs. Nevertheless, current EAU guidelines recommend the use of antimuscarinics in males with moderate-to-severe LUTS with a primary storage component, but prescription should be done with caution and regular reevaluation of males with $\mathrm{BOO}$ is highly recommended. ${ }^{18}$

\section{$\beta_{3}$-adrenergic agonists}

While $\alpha$-adrenoceptors are expressed in high numbers in prostatic stromal cells, $\beta$-adrenoceptors dominate in the bladder, and their activation induces bladder relaxation during the storage phase of the micturition cycle. ${ }^{29}$ There are three subtypes of $\beta$-adrenoceptors $-\beta_{1}, \beta_{2}$, and $\beta_{3}$-with $\beta_{3}$-receptors the predominant type in human detrusor muscle. ${ }^{29} \beta_{3}$-Adrenoceptors can also be found in the urothelial and interstitial cells of the bladder, however, their exact functional role remains to be determined. ${ }^{30}$ Several experimental studies have shown that activation of $\beta_{3}$-receptors leads to increased bladder compliance, without influence on detrusor contraction during the voiding phase or increase in PVR ${ }^{31}$ Currently, only mirabegron, a selective $\beta_{3}$-adrenergic receptor agonist, is available for use in males with OAB symptoms.

Several studies have assessed the effectiveness of mirabegron use in male patients with OAB symptoms. Large controlled studies showed that mirabegron use led to significant improvement in symptoms, such as frequency, urgency, nocturia, and incontinence, as well as in general parameters tested, including QoL, patient perception of bladder condition, and satisfaction with treatment. ${ }^{32}$ Mirabegron showed a significant response in symptoms, even if patients had a history of use of antimuscarinics that was discontinued because of unsatisfactory outcomes or safety issues. In the same group of patients, retreatment with tolterodine led to improvement in symptoms in males who discontinued antimuscarinics as a result of side effects, but not in males who stopped treatment with antimuscarinics because of insufficient efficacy. ${ }^{33,34}$

Mirabegron use has been proved to be relatively safe, as acceptable rates of adverse events and withdrawal rates have been reported in several clinical trials. ${ }^{35,36}$ Hypertension, nasopharyngitis, and urinary tract infections comprise the main side effects related to mirabegron use, while no difference was found in comparison to placebo in dry mouth or constipation, which represent the main complications associated with antimuscarinic use. ${ }^{35,36}$ It has to be noted that due to reported events of severe hypertension in patients receiving mirabegron, it is advised that blood pressure is measured and regularly monitored prior to onset of and during treatment, as poorly controlled hypertension is a main contraindication for mirabegron use. ${ }^{37}$ As mirabegron is an inhibitor of CYP isoenzymes 2D6 and 3A, concomitant administration with drugs that interact with CYP, such as desipramine, rifampin, and metoprolol, should be done with caution. ${ }^{30}$

In males with combined $\mathrm{BOO}$ and $\mathrm{OAB}$, mirabegron did not adversely affect voiding urodynamic parameters compared to placebo in terms of $\mathrm{Q}_{\max }$, detrusor pressure at maximum flow, or bladder-contractility index, while minor increases in PVR were reported in patients under treatment with $100 \mathrm{mg}$ of mirabegron versus no effect in those treated with $50 \mathrm{mg} \cdot{ }^{38}$ Based on the new evidence, $\beta_{3}$-agonists may be used in males with moderate-to-severe LUTS with a predominant storage component.

\section{Phosphodiesterase-5 inhibitors}

Erectile dysfunction and BPH seem to share common risk factors that include age, obesity, metabolic disorders such as diabetes mellitus and hypogonadism, and cardiovascular disorders such as hypertension. ${ }^{39}$ Suggested pathogenetic pathways comprise impaired nitric oxide and cyclic guanosine monophosphate signaling, increased signaling of RhoA/ Rho kinase, autonomic adrenergic overactivity, increased afferent activity to the bladder and prostate, and pelvic ischemia due to atherosclerosis. ${ }^{40}$ Therapeutic actions of phosphodiesterase-5 inhibitors (PDE5Is) have been reported to be implicated in all these pathways, and effects of PDE5Is on the bladder, prostate, pelvic vasculature, and even spinal cord have been demonstrated. ${ }^{40-42}$ 
Several reports on various PDE5I use for male LUTS have been published, and have shown beneficial effects for sildenafil, vardenafil, tadalafil, and UK-369003. ${ }^{40,43}$ PDE5Is have been used as monotherapies or in combination with $\alpha_{1}$-blockers. ${ }^{40,43}$ Compared to placebo, monotherapy with PDE5Is was proved to improve IPSS significantly at the end of several trials, while combination treatment has been proved to be better than monotherapy with $\alpha_{1}$-blockers in terms of IPSS improvement and $\mathrm{Q}_{\max }$ increase. ${ }^{40}$ Interestingly, males of younger age, with lower body mass index, and severer degree of LUTS seem to benefit the most from PDE5I use. It has been proposed that older males and males with higher body mass index have lower testosterone levels that lead to decreased expression of PDE5 in the bladder and thus to more unfavorable treatment outcome. ${ }^{40}$

As expected, and in contrast to other treatment modalities for LUTS, PDE5Is induce beneficial effects in erectile function parameters as well. An overall incidence of treatmentrelated adverse events of $16 \%$ has been shown, mainly of mild-to-moderate degree of severity. Flushing, headache, gastroesophageal reflux, dyspepsia, back pain, and nasal congestion have been reported as common side effects of PDE5I use. ${ }^{40}$ Several meta-analyses of available studies on PDE5I use in males with BPH-related LUTS highlight their heterogeneity, their relatively short duration, and the risk of bias. While no difference in treatment effect seems to exist among the various PDE5Is (even though no direct comparative randomized controlled trials [RCTs] are currently available), to date only tadalafil at a daily dose of $5 \mathrm{mg}$ has been licensed for managing LUTS in males suffering from BPH with or without concomitant disorders of erectile function.

\section{$\alpha_{1}$-Blockers $+5 \alpha$-reductase inhibitors}

Representing the currently most popular treatment modalities for BPH-related LUTS, $\alpha_{1}$-blockers and $5 \alpha$ RIs have different modes of action, and thus a synergistic effect can be expected with their combined use. Several trials have used various combinations of $\alpha_{1}$-blockers and $5 \alpha$ RIs, and currently available long-term data reveal superior effects of combination treatment over $\alpha_{1}$-blocker monotherapies in improving symptom severity and selected urodynamic parameters. ${ }^{14,15}$ With regard to prevention or delay of BPH progression in terms of AUR episodes or need for surgical management, different findings have been reported by two large studies using different combinations and different methodology. The MTOPS study used doxazosin and finasteride, and its findings demonstrated that combination treatment had significantly better results in reducing the risk of AUR or need for surgery. ${ }^{14}$ The CombAT trial, on the other hand, used a combination of tamsulosin and dutasteride, and showed that combination treatment was superior to either monotherapy in inhibiting progression of BPH. ${ }^{15}$ Recently, the CONDUCT study, which was an international, multicenter, randomized, open-label, parallel-group, Phase IV study, investigated whether immediate therapy with a fixed-dose combination (FDC) of $0.5 \mathrm{mg}$ dutasteride and $0.4 \mathrm{mg}$ tamsulosin in treatment-naïve males with moderate LUTS and risk factors for BPH progression offered faster and more profound symptom reduction than that achieved by watchful waiting plus initiation of tamsulosin if symptoms did not improve. Both groups received lifestyle advice on dietary habits, fluid management, and bladder-training exercises. This study verified the therapeutic effects of combination treatment in terms of symptom improvements and reduction in relative risk for clinical progression. Sustained efficacy of FDC therapy over the 2-year follow-up and satisfaction with its use was also found. ${ }^{44}$

A synergistic effect in adverse events was also revealed by studies evaluating combination treatments, though. Higher rates of adverse events were reported by males under combination treatment versus either monotherapy, with the majority of them complaining mainly of sexual disorders, especially erectile and ejaculatory dysfunction. ${ }^{14,15,44}$ Overall, and based on current evidence, EAU guidelines state that long-term combination treatment with $\alpha_{1}$-blockers and $5 \alpha$ RIs can be offered to males with BPH-related LUTS of moderate-tosevere degree, high prostatic volume, and decreased maximum urinary flow, who represent the group of patients most likely to develop BPH progression. ${ }^{18}$

\section{$\alpha_{1}$-Blockers + muscarinic receptor antagonists}

A reasonable approach to alleviate symptoms and improve patients' QoL in males with predominant storage symptoms, but also with voiding symptoms, would be to combine medications that aim at the prostate and the bladder at the same time. Therefore, $\alpha_{1}$-blockers have been used in conjunction with muscarinic receptor antagonists as either initial or add-on therapy if symptoms were not fully managed by either monotherapy. Results from available trials have shown beneficial effects by combination treatment in urgency, frequency, nocturia, and urgency-incontinence episodes versus monotherapy with $\alpha_{1}$-blockers. ${ }^{7,18}$ Adding an antimuscarinic agent to manage residual LUTS despite initial treatment with an $\alpha_{1}$-blocker has also been shown to lead to positive outcomes, especially if the storage component of LUTS persisted. An FDC tablet containing tamsulosin as $\alpha_{1}$-blocker and solifenacin 
as antimuscarinic has been released for use in males with moderate-to-severe storage and voiding BPH-related LUTS not adequately responding to initial treatment with either monotherapy. Results from available studies confirm improvement in symptoms and QoL compared to monotherapy, as shown in research using two separate agents. ${ }^{25}$

Since two different modes of treatment are combined, a higher incidence of adverse events is to be expected. In general, combination treatment of any type (separate administration or FDC tablet) has been proved to be safe, with dry mouth and ejaculation disorders being the most frequently reported adverse events, mainly of mild-to-moderate degree. ${ }^{7}$ A slight and clinically insignificant increase in PVR has been observed in patients receiving combination treatment with $\alpha_{1}$-blockers and antimuscarinics, but the AUR rate was low. However, most studies have received criticism on their selection criteria, end points, and short duration, which contrasts with daily clinical practice in males with LUTS. Combination treatment in males with bothersome moderate-to-severe LUTS is recommended if relief of storage symptoms has been insufficient with monotherapy with either drug. Still, physicians' alertness is highlighted, as despite insignificant PVR increase and low AUR rates, combination treatment is recommended with caution in males with possible BOO. ${ }^{18}$

\section{Treatment modalities currently under investigation Anti-inflammatory agents}

The role of chronic inflammation in BPH has been explored by several studies. ${ }^{32} \mathrm{~A}$ strong correlation between $\mathrm{BPH}$ and inflammation has been shown, despite the fact that severity of inflammation cannot predict the severity of symptoms reported by patients. ${ }^{45}$ Therefore, agents that mediate inflammation could have a therapeutic potential in BPH management, even though response in LUTS could be expected to be minimal. Conflicting results from several studies evaluating the role of anti-inflammatory drugs on BPH and LUTS are available in the literature, and thus clear recommendations cannot be drawn. ${ }^{46}$ Moreover, a lot of available studies are characterized by their relatively short duration and lack of randomization and control group, and as such no high-quality data can be extracted from their analysis. ${ }^{32}$

\section{Vitamin $D_{3}$-receptor analogs}

Among several receptors expressed in the lower urinary tract, vitamin $\mathrm{D}_{3}$ receptors in the bladder, prostate, and prostatic urethra have also been studied, as their activation seems to inhibit proliferation and induce apoptosis of prostatic stromal cells, and mediate signaling in bladder-muscle cells. ${ }^{32}$ Elocalcitol, a vitamin $\mathrm{D}_{3}$-receptor agonist, has been evaluated in several studies, and encouraging findings were presented. In particular, elocalcitol use in rats led to fewer spontaneous bladder contractions, despite no effect being shown on bladder hypertrophy. ${ }^{47}$ Also in rats, better results on bladder compliance from tolterodine administration were presented if prior treatment with elocalcitol took place. ${ }^{48}$ In human patients $\geq 50$ years of age with prostate volumes $\geq 40 \mathrm{cc}^{3}$, elocalcitol has been proved to arrest further prostate growth, but no improvement in reported symptoms and maximum urinary flow rate was found, thus questioning the role of elocalcitol in LUTS management. ${ }^{49}$ Recently, OAB females participating in a Phase II RCT were treated with elocalcitol and reported fewer incontinence episodes and better perception of bladder condition, but unfortunately no male patients were evaluated in this trial. ${ }^{50}$

\section{Cannabinoids}

Studies conducted in patients with multiple sclerosis and LUTS have shown beneficial effects in urgency, frequency, nocturia, and incontinence episodes with treatment using cannabinoids, as the cannabinoid system has been proposed to participate in the control of micturition. ${ }^{46}$ Various degrees of effect have been shown in cannabinoid treatment though, as results from a multicenter RCT demonstrated significantly fewer episodes of incontinence with $\Delta^{9}$-tetrahydrocannabinol administration to multiple sclerosis patients, while a study that used a modulator of the endocannabinoid system (nabiximols), reported fewer daily and daytime voids and improved nocturia in the active-treatment group, but no significant effects on incontinence episodes, which represented the study's primary end point. ${ }^{51,52}$ To date, there have been no clinical studies evaluating the role of cannabinoid use in males with nonneurogenic LUTS, and as such cannabinoid administration to them can be regarded as experimental.

\section{Luteinizing hormone-releasing hormone antagonist}

Cetrorelix is a luteinizing hormone-releasing hormone (LHRH) antagonist that has been tested for BPH management. LHRH antagonists can have therapeutic effects on male LUTS, as they offer intermediate systemic suppression of testosterone without the severe adverse events of medical castration caused by antiandrogens or LHRH agonists. ${ }^{53}$ In a Phase II study, intramuscular injection of cetrorelix $60 \mathrm{mg}$ led to a 6-month sustained 4-point reduction in IPSS versus placebo administration, an effect, however, that was not observed in Phase III RCTs that followed. ${ }^{54,55}$ 


\section{Other treatments under investigation}

Various transient receptor potential (TRP) channels have been proposed to respond to stretch and/or chemical irritation, and thus have been suggested to participate in the pathogenetic mechanisms of LUTS. ${ }^{32}$ Specifically, the TRPM8, TRPV1, TRPC1, TRPC4, TRPA1, and TRPV4 channels have been described to be involved in pathways of bladder dysfunction and related symptoms. ${ }^{56}$ Even though an adequate amount of literature is now available, the exact way that malfunction of TRP channels mediates bladder pathogenesis has yet to be determined. Several studies conducted in animals have tested the role of specific TRP-channel blockers, and have shown encouraging results in terms of bladder-overactivity parameters. ${ }^{32,46}$ However, due to hyperthermic effects, there are no available studies evaluating the role of TRP-channel inhibitors in alleviating LUTS in males with BPH. Nevertheless, manipulation of TRP channels to alleviate symptoms of bladder dysfunction remains an extremely interesting concept of treatment for LUTS.

Moreover, several studies have revealed the role of purinergic neurotransmission in the bladder urothelium and bladder sensory nerve fibers, especially in pathologic bladder conditions, such as those deriving from BOO. In general, activation of purinoceptors has a limited role in normal bladder function; however the role of purinergic neurotransmission becomes evident and clinically significant in bladder dysfunction. Distended bladder tissue releases ATP that acts on the $\mathrm{P} 2 \mathrm{X} 3$ and $\mathrm{P} 2 \mathrm{X} 2 / 3$ receptors, which subsequently trigger the voiding reflex and nociception. ${ }^{57}$ Various antagonists of purinergic receptors have been tested in animals, but none of them has been tested in clinical setting in human male patients. $^{32,46}$

Likewise, several Rho-kinase inhibitors have been tested in animals with bladder dysfunction and shown satisfactory results, but no similar tests have been conducted in human patients. ${ }^{32}$ In addition, endothelin 1 has been proved to control contraction of the human detrusor, and it has been shown to be overexpressed in the detrusor muscle of rabbits with $\mathrm{BOO}$. Endothelin 1 is generated by ECE, and thus inhibitors of ECE could mediate endothelin 1-induced bladder contractions. To date, no clinical trials using ECE inhibitors in males with LUTS have been performed. ${ }^{32}$

\section{Strategies for optimal selection of medical treatment for male LUTS}

The desired goals of medical treatment of male LUTS include durable, clinically significant improvement in symptoms and QoL and/or slowing or preventing the progression of the condition. This review shows that several therapeutic options are currently available for medical management of male LUTS. The increasing understanding of the underlying pathophysiological mechanisms and natural history of LUTS dictate our therapeutic strategies. Stratification of patients according to their presenting symptoms becomes imperative for selection of proper treatment. Besides symptoms, other factors that can affect selection of therapeutic modality include patients' medical history and comorbidities, status of sexual functioning, patients' preference, and objective measurements derived from clinical and laboratory evaluation, such as prostatic volume, PVR volume, PSA, and IPSS. ${ }^{58}$ Recent evidence supports the multifactorial pathogenesis of this condition, indicating that a combination of available drug classes should also be used.

Education and lifestyle modification should be offered to all patients, regardless of the severity or quality of their symptoms. ${ }^{18}$ The level of bother is crucial for the treatment of choice, as it has been proved that males with mild-tomoderate LUTS and favorable clinical parameters who are minimally bothered by their symptoms can be effectively managed by watchful waiting and proposed modifications in lifestyle. ${ }^{18,59}$

Medical treatment should be offered to patients with bothersome symptoms. The type of symptoms will guide our initial therapeutic strategy. If storage LUTS represent the main bothersome symptoms reported by patients with no evidence of BPE/BPH-related BOO, bladder-targeting drugs, such as antimuscarinics or $\beta_{3}$-agonists, can be prescribed, if behavioral modifications fail to control complaints. ${ }^{18}$ However, if prostatic obstruction is suspected, patients should be offered combined treatment with $\alpha_{1}$-blockers and antimuscarinics or $\beta_{3}$-agonists.

Voiding symptoms seem to be directly related to the prostate, and thus evaluation of prostatic volume, maximum urinary flow, PVR volume, and PSA value are needed to identify patients at risk for disease progression. ${ }^{58}$ Patients with a prostate volume $<40 \mathrm{~cm}^{3}$ and predominant voidingbothersome LUTS are managed with $\alpha_{1}$-blockers. In the case of persisting storage symptoms despite $\alpha_{1}$-blocker therapy, adding antimuscarinics or $\beta_{3}$-agonists seems a reasonable approach to manage the whole LUTS spectrum properly. In contrast, patients at risk for disease progression are more likely to benefit from a combined treatment with a $5 \alpha \mathrm{RI}$ and an $\alpha_{1}$-blocker if patients are willing to undergo the longlasting treatment required for $5 \alpha$ RIs.

With respect to PDE5Is, they can be offered to males with moderate-to-severe voiding and storage LUTS, regardless of the presence or absence of concomitant erectile dysfunction. 
However, it seems that in males with erectile dysfunction and LUTS, tadalafil $5 \mathrm{mg}$ once a day represents the most sensible therapeutic option in an effort to reduce symptoms and manage sexual dysfunction at the same time, without the sexual adverse events of other medical therapies. It is useful for daily clinical practice to remember that younger males with a low body mass index and severe LUTS seem to be those more likely to benefit from PDE5I use.

In addition, the present review showed that there are several new therapies under investigation in trials that may be emerging in the management of LUTS. The reasons for this continuous interest in research include the high prevalence of the condition and the rather unmet expectations of patients and physicians regarding the efficacy of available therapies. Table 1 presents the current status supporting the use of the available medications for the management of male LUTS.

\section{Conclusion}

As LUTS are multifactorial in origin and diverse in presentation, a one-for-all treatment does not seem appropriate. New evidence emphasizing the bladder's role in pathogenesis of LUTS questions traditional therapies aiming solely at the prostate, such as $\alpha_{1}$-blockers and $5 \alpha$ RIs. The role of antimuscarinics and $\beta_{3}$-agonists in alleviating the highly bothersome storage

Table I Status of the available medical options for management of male LUTS

\begin{tabular}{ll}
\hline Treatment option & Status \\
\hline$\alpha_{1}$-Blockers & Established \\
$5 \alpha$-RIs & Established \\
Antimuscarinics & Established \\
PDE5ls* & Established \\
$\beta_{3}$-Agonists & Emerging \\
Combination $\alpha_{1}$-Blockers $+5 \alpha$-Rls & Established \\
Combination $\alpha_{1}$-Blockers + antimuscarinics & Established \\
Anti-inflammatory & Experimental \\
Vitamin $D_{3}$-receptor analogs & Experimental \\
LHRH antagonists & Experimental \\
Cannabinoids & Experimental \\
TRP-channel blockers & \\
Antagonists of purinergic receptors & Experimental in animal \\
Rho-kinase inhibitors & setting \\
Inhibitors of ECE & Experimental in animal \\
& setting \\
\hline
\end{tabular}

Note: *Only tadalafil $5 \mathrm{mg}$ has been licensed.

Abbreviations: LUTS, lower urinary tract symptoms; RIs, reductase inhibitors; PDE5ls, phosphodiesterase-5 inhibitors; LHRH, luteinizing-hormone releasing hormone; TRP, transient receptor potential; ECE, endothelin-converting enzyme. component of male LUTS now becomes highlighted, despite the initial reluctance of urologists in their use. Various combination treatments are encouraged in current guidelines, as they have been proved to offer superior synergistic effects in managing patients' complaints in comparison to monotherapies. PDE5Is now offer not only beneficial effects on male sexual function, but in voiding and storage LUTS too, as an alternative to $\alpha_{1}$-blockers. Furthermore, many potential targets for future drugs have been evaluated, and new agents are currently under investigation or in the clinical trial setting to enhance the armamentarium of therapeutic modalities for male LUTS further.

\section{Disclosure}

SG has received consulting fees, honoraria, and research support from Pierre Fabre Medicament, GlaxoSmithKline, Astellas, Angelini Pharma Hellas, and Lilly. KD reports no conflicts of interest in this work.

\section{References}

1. Abrams P, Cardozo L, Fall M, et al. The standardisation of terminology in lower urinary tract function: report from the Standardisation Sub-committee of the International Continence Society. Urology. 2003;61(1):37-49.

2. Chapple CR, Wein AJ, Abrams P, et al. Lower urinary tract symptoms revisited: a broader clinical perspective. Eur Urol. 2008;54(3):563-569.

3. Chapple, C, Abrams P. Lower Urinary Tract Symptoms (LUTS): An International Consultation on Male LUTS. Montreal: Société Internationale d'Urologie; 2013.

4. Martin SA, Haren MT, Marshall VR, Lange K, Wittert GA, Members of the Florey Adelaide Male Ageing Study. Prevalence and factors associated with uncomplicated storage and voiding lower urinary tract symptoms in community-dwelling Australian men. World J Urol. 2011;29(2):179-184.

5. Kupelian V, Wei JT, O'Leary MP, et al. Prevalence of lower urinary tract symptoms and effect on quality of life in a racially and ethnically diverse random sample: the Boston Area Community Health (BACH) Survey. Arch Intern Med. 2006;166(21):2381-2387.

6. Irwin DE, Milsom I, Hunskaar S, et al. Population-based survey of urinary incontinence, overactive bladder, and other lower urinary tract symptoms in five countries: results of the EPIC study. Eur Urol. 2006;50(6):1306-1314; discussion 1314-1315.

7. Füllhase C, Chapple C, Cornu J-N, et al. Systematic review of combination drug therapy for non-neurogenic male lower urinary tract symptoms. Eur Urol. 2013;64(2):228-243.

8. Abrams P. Benign prostatic hyperplasia: poorly correlated with symptoms. BMJ. 1993;307(6897):201.

9. Dmochowski RR, Staskin D. Overactive bladder in men: special considerations for evaluation and management. Urology. 2002;60(5 Suppl 1): 56-62; discussion 62-63.

10. Wasson JH, Reda DJ, Bruskewitz RC, Elinson J, Keller AM, Henderson WG. A comparison of transurethral surgery with watchful waiting for moderate symptoms of benign prostatic hyperplasia. The Veterans Affairs Cooperative Study Group on Transurethral Resection of the Prostate. N Engl J Med. 1995;332(2):75-79.

11. Flanigan RC, Reda DJ, Wasson JH, Anderson RJ, Abdellatif M, Bruskewitz RC. 5-Year outcome of surgical resection and watchful waiting for men with moderately symptomatic benign prostatic hyperplasia: a Department of Veterans Affairs cooperative study. J Urol. 1998;160(1):12-16; discussion 16-17. 
12. Roosen A, Chapple CR, Dmochowski RR, et al. A refocus on the bladder as the originator of storage lower urinary tract symptoms: a systematic review of the latest literature. Eur Urol. 2009;56(5):810-819.

13. Chapple CR, Roehrborn CG. A shifted paradigm for the further understanding, evaluation, and treatment of lower urinary tract symptoms in men: focus on the bladder. Eur Urol. 2006;49(4):651-658.

14. McConnell JD, Roehrborn CG, Bautista OM, et al. The long-term effect of doxazosin, finasteride, and combination therapy on the clinical progression of benign prostatic hyperplasia. $N$ Engl J Med. 2003;349(25):2387-2398.

15. Roehrborn CG, Siami P, Barkin J, et al. The effects of combination therapy with dutasteride and tamsulosin on clinical outcomes in men with symptomatic benign prostatic hyperplasia: 4-year results from the CombAT study. Eur Urol. 2010;57(1):123-131.

16. Kortmann BBM, Floratos DL, Kiemeney LALM, Wijkstra H, de la Rosette JJMCH. Urodynamic effects of alpha-adrenoceptor blockers: a review of clinical trials. Urology. 2003;62(1):1-9.

17. Barendrecht MM, Abrams P, Schumacher H, de la Rosette JJMCH, Michel MC. Do alpha1-adrenoceptor antagonists improve lower urinary tract symptoms by reducing bladder outlet resistance? Neurourol Urodyn. 2008;27(3):226-230.

18. Oelke M, Bachmann A, Descazeaud A, et al. EAU guidelines on the treatment and follow-up of non-neurogenic male lower urinary tract symptoms including benign prostatic obstruction. Eur Urol. 2013;64(1):118-140.

19. Naslund MJ, Miner M. A review of the clinical efficacy and safety of $5 \alpha$-reductase inhibitors for the enlarged prostate. Clin Ther. 2007;29(1):17-25.

20. Nickel JC, Fradet Y, Boake RC, et al. Efficacy and safety of finasteride therapy for benign prostatic hyperplasia: results of a 2-year randomized controlled trial (the PROSPECT study). PROscar Safety Plus Efficacy Canadian Two year Study. CMAJ. 1996;155(9):1251-1259.

21. Roehrborn CG, Boyle P, Nickel JC, Hoefner K, Andriole G, ARIA3001 ARIA3002 and ARIA3003 Study Investigators. Efficacy and safety of a dual inhibitor of 5-alpha-reductase types 1 and 2 (dutasteride) in men with benign prostatic hyperplasia. Urology. 2002;60(3):434-441.

22. Chughtai B, Elterman DS, Lee R, Te AE, Kaplan SA. Experience with the combination of dutasteride and tamsulosin in the longterm management of benign prostatic hyperplasia. Ther Adv Urol. 2012;4(5):267-272.

23. Sexton CC, Coyne KS, Kopp ZS, et al. The overlap of storage, voiding and postmicturition symptoms and implications for treatment seeking in the USA, UK and Sweden: EpiLUTS. BJU Int. 2009;103(Suppl 3): 12-23.

24. Peters TJ, Donovan JL, Kay HE, et al. The International Continence Society "Benign Prostatic Hyperplasia" Study: the botherosomeness of urinary symptoms. J Urol. 1997;157(3):885-889.

25. Dimitropoulos K, Gravas S. Solifenacin/tamsulosin fixed-dose combination therapy to treat lower urinary tract symptoms in patients with benign prostatic hyperplasia. Drug Des Devel Ther. 2015;9:1707-1716.

26. Kaplan SA, Wein AJ, Staskin DR, Roehrborn CG, Steers WD. Urinary retention and post-void residual urine in men: separating truth from tradition. J Urol. 2008;180(1):47-54.

27. Kaplan SA, Walmsley K, Te AE. Tolterodine extended release attenuates lower urinary tract symptoms in men with benign prostatic hyperplasia. J Urol. 2005;174(6):2273-2275. discussion 2275-2276.

28. Yokoyama T, Uematsu K, Watanabe T, et al. Naftopidil and propiverine hydrochloride for treatment of male lower urinary tract symptoms suggestive of benign prostatic hyperplasia and concomitant overactive bladder: a prospective randomized controlled study. Scand J Urol Nephrol. 2009;43(4):307-314.

29. Yamaguchi O. Latest treatment for lower urinary tract dysfunction: therapeutic agents and mechanism of action. Int J Urol. 2013;20(1):28-39.

30. Vij M, Drake MJ. Clinical use of the $\beta 3$ adrenoceptor agonist mirabegron in patients with overactive bladder syndrome. Ther Adv Urol. 2015;7(5):241-248.
31. Kanai A, Wyndaele J-J, Andersson K-E, et al. Researching bladder afferents-determining the effects of $\beta(3)$-adrenergic receptor agonists and botulinum toxin type-A. Neurourol Urodyn. 2011;30(5): 684-691.

32. Soler R, Andersson K-E, Chancellor MB, et al. Future direction in pharmacotherapy for non-neurogenic male lower urinary tract symptoms. Eur Urol. 2013;64(4):610-621.

33. Füllhase C, Soler R, Gratzke C. New strategies in treating male lower urinary tract symptoms. Curr Opin Urol. 2014;24(1):29-35.

34. Schauer I, Madersbacher S. Medical treatment of lower urinary tract symptoms/benign prostatic hyperplasia: anything new in 2015. Curr Opin Urol. 2015;25(1):6-11.

35. Khullar V, Amarenco G, Angulo JC, et al. Efficacy and tolerability of mirabegron, a $\beta(3)$-adrenoceptor agonist, in patients with overactive bladder: results from a randomised European-Australian phase 3 trial. Eur Urol. 2013;63(2):283-295.

36. Chapple CR, Kaplan SA, Mitcheson D, et al. Randomized double-blind, active-controlled phase 3 study to assess 12-month safety and efficacy of mirabegron, a $\beta(3)$-adrenoceptor agonist, in overactive bladder. Eur Urol. 2013;63(2):296-305.

37. EUROPEAN MEDICINES AGENCY: Summary of product characteristics. http://www.ema.europa.eu/docs/en_GB/document_library/ EPAR_-_Product_Information/human/002388/WC500137309.pdf. Accessed, March 14, 2016

38. Nitti VW, Auerbach S, Martin N, Calhoun A, Lee M, Herschorn S. Results of a randomized phase III trial of mirabegron in patients with overactive bladder. J Urol. 2013;189(4):1388-1395.

39. Haddad A, Jabbour M, Bulbul M. Phosphodiesterase type 5 inhibitors for treating erectile dysfunction and lower urinary tract symptoms secondary to benign prostatic hyperplasia: a comprehensive review. Arab J Urol. 2015;13(3):155-161.

40. Gacci M, Corona G, Salvi M, et al. A systematic review and metaanalysis on the use of phosphodiesterase 5 inhibitors alone or in combination with $\alpha$-blockers for lower urinary tract symptoms due to benign prostatic hyperplasia. Eur Urol. 2012;61(5):994-1003.

41. Lythgoe C, McVary KT. The use of PDE-5 inhibitors in the treatment of lower urinary tract symptoms due to benign prostatic hyperplasia. Curr Urol Rep. 2013;14(6):585-594.

42. Gratzke C, Hennenberg M, Stief C. Pharmacology of the lower urinary tract. Indian Journal of Urology. 2014;30(2):181-188.

43. Wang X-H, Wang X, Zhang X-H, Li S, Liu T, Shi M-J. Systematic review and meta-analysis on phosphodiesterase 5 inhibitors and $\alpha$-adrenoceptor antagonists used alone or combined for treatment of LUTS due to BPH. Asian Journal of Andrology. 2015;17(6):1022-1032.

44. Roehrborn CG, Oyarzabal Perez I, Roos EPM, et al. Efficacy and safety of a fixed-dose combination of dutasteride and tamsulosin treatment (Duodart $\left({ }^{\circledR}\right)$ ) compared with watchful waiting with initiation of tamsulosin therapy if symptoms do not improve, both provided with lifestyle advice, in the management of treatment-naïve men with moderately symptomatic benign prostatic hyperplasia: 2-year CONDUCT study results. BJU Int. 2015;116(3):450-459.

45. Nickel JC, Roehrborn CG, O’Leary MP, Bostwick DG, Somerville MC, Rittmaster RS. The relationship between prostate inflammation and lower urinary tract symptoms: examination of baseline data from the REDUCE trial. Eur Urol. 2008;54(6):1379-1384.

46. Füllhase $\mathrm{C}$, Hakenberg $\mathrm{O}$. New concepts for the treatment of male lower urinary tract symptoms. Curr Opin Urol. 2015;25(1):19-26.

47. Schröder A, Colli E, Maggi M, Andersson K-E. Effects of a vitamin D3 analogue in a rat model of bladder outlet obstruction. BJU International. 2006;98(3):637-642.

48. Streng T, Andersson K-E, Hedlund P, et al. Effects on bladder function of combining elocalcitol and tolterodine in rats with outflow obstruction. BJU International. 2012;110(2b):E125-E131.

49. Tiwari A. Elocalcitol, a vitamin D3 analog for the potential treatment of benign prostatic hyperplasia, overactive bladder and male infertility. IDrugs. 2009;12(6):381-393. 
50. Digesu GA, Verdi E, Cardozo L, Olivieri L, Khullar V, Colli E. Phase IIB, multicenter, double-blind, randomized, placebo-controlled, parallel-group study to determine effects of elocalcitol in women with overactive bladder and idiopathic detrusor overactivity. Urology. 2012;80(1):48-54

51. Freeman RM, Adekanmi O, Waterfield MR, Waterfield AE, Wright D, Zajicek $\mathrm{J}$. The effect of cannabis on urge incontinence in patients with multiple sclerosis: a multicentre, randomised placebo-controlled trial (CAMS-LUTS). Int Urogynecol J. 2006;17(6):636-641.

52. Brady CM, DasGupta R, Dalton C, Wiseman OJ, Berkley KJ, Fowler CJ. An open-label pilot study of cannabis-based extracts for bladder dysfunction in advanced multiple sclerosis. Mult Scler. 2004;10(4):425-433.

53. Lepor $\mathrm{H}$. The role of gonadotropin-releasing hormone antagonists for the treatment of benign prostatic hyperplasia. Rev Urol. 2006;8(4): 183-189.

54. Debruyne F, Tzvetkov M, Altarac S, Geavlete PA. Dose-ranging study of the luteinizing hormone-releasing hormone receptor antagonist cetrorelix pamoate in the treatment of patients with symptomatic benign prostatic hyperplasia. Urology. 2010;76(4):927-933.
55. Daly DM, Collins VM, Chapple CR, Grundy D. The afferent system and its role in lower urinary tract dysfunction. Curr Opin Urol. 2011;21(4):268-274.

56. Franken J, Uvin P, De Ridder D, Voets T. TRP channels in lower urinary tract dysfunction. Br J Pharmacol. 2014;171(10):2537-2551.

57. Burnstock G. Purinergic signalling in the urinary tract in health and disease. Purinergic Signal. 2014;10(1):103-155.

58. Gratzke C, Bachmann A, Descazeaud A, et al. EAU guidelines on the assessment of non-neurogenic male lower urinary tract symptoms including benign prostatic obstruction. Eur Urol. 2015;67(6):1099-1109.

59. Kirby RS. The natural history of benign prostatic hyperplasia: what have we learned in the last decade? Urology. 2000;56(5 Suppl 1):3-6.
Research and Reports in Urology

\section{Publish your work in this journal}

Research and Reports in Urology is an international, peer-reviewed, open access journal publishing original research, reports, editorials, reviews and commentaries on all aspects of adult and pediatric urology in the clinic and laboratory including the following topics: Pathology, pathophysiology of urological disease; Investigation and treatment of

\section{Dovepress}

urological disease; Pharmacology of drugs used for the treatment of urological disease. The manuscript management system is completely online and includes a very quick and fair peer-review system, which is all easy to use. Visit http://www.dovepress.com/testimonials.php to read real quotes from published authors.

Submit your manuscript here: http://www.dovepress.com/research-and-reports-in-urology-journal 